\title{
Textwall- Integrated IT intervention or irritating, irrelevant intrusion
}

Author: Bob Morris. Liverpool John Moores University, Liverpool

Background: Textwall by Learning Apps is a technological tool available to encourage participants in large or medium sized groups to engage with speakers and their delivered content using phones, email or via the internet. Students are invited to send messages during lectures that can either be incorporated into the lecture content or discussed following the lecture. These could be in response to a question posed by the lecturer or generated from students' considering the lecture content. By involving students in lectures more it may be possible to integrate the content of the individual session with other parts of their whole learning experience. Academics delivering material that may be considered as uninspiring may be interested in this study to help improve their students' experience and also achieve learning outcomes more effectively.

Description of work: Following encouraging demonstrations at post graduate and non-university events I was tempted to embrace the technology as high value is placed in Higher Education on student engagement. The study aims to identify the types of responses generated by pharmacy undergraduates at one UK school of pharmacy to inform use of Textwall in delivering engaging lectures. Responses will be categorised into similar themes and an investigation of the different ways responses are used in teaching will be undertaken. Experiences and possible learnings will be reported. Throughout 2013-14 I have used Textwall in most of my large group teaching undergraduate and postgraduate sessions. I identified some benefits and drawbacks immediately so decided to record and categorise responses. I have performed a simple quantitative analysis of this data and will report results of this analysis.

Possible Evaluation: Quantitative and qualitative data will be analysed. Following categorisation of responses and source of comments types of responses will be compared to identify areas to improve the delivery of pharmacy lectures. Suggested use of Textwall will be identified from responses. 